\title{
Enhancing Gradient Sparsity for Parametrized Motion Estimation
}

\author{
Junyu Han \\ pengjie.han@gmail.com \\ Fei Qi \\ fred.qi@ieee.org \\ Guangming Shi \\ gmshi@xidian.edu.cn
}

School of Electronic Engineering

Xidian University

Xi'an, Shaanxi, P. R. China

\begin{abstract}
In this paper, we propose a novel motion estimation framework based on the sparsity associated with gradients of the parametrized motion field. Beginning with Shen and Wu's sparse model for optic flow estimation [ㅁ], we show the sparsity of the motion field can be enhanced by increasing the degree of freedom of the parametrized motion model. With such an enhancement, we formulate the motion estimation as an $\ell_{0}$ optimization problem. Along with an $\ell_{1}$ norm regularization to the instant constancy assumption, this problem is solved by a reweighted $\ell_{1}$ optimization approach. Experiments on constant, pure translational, and affine motion models certify that the enhanced sparsity provides improved accuracy for motion estimation.
\end{abstract}

\section{Introduction}

Optic flow extracts motion information from image sequences. It is very important for video processing, analysis, and understanding, and is widely used in such applications as video frame rate conversion, structure from motion, object tracking, and behavior analysis, etc.

Most existing optic flow estimation approaches are based on the constant intensity assumption which deduces an under-determined linear system. To solve this ill-posed problem, following either the Lucas-Kanade local model [ $\square]$ or Horn-Schunck global model [ $[$ ] many concepts and methods $[\square, \square]$ were proposed. These methods perform well when the prerequisites employed hold. However, motion boundaries, where most of these prerequisites break, are widely exist due to the complex textures and shapes of objects and the coexistence of multiple moving objects.

To deal with discontinuities on motion boundaries, various regularization schemes for the variational model were proposed. Black and Anandan introduced a robust penalization instead of the square error [ []]. Sun et al. introduced an approach to inference the flow filed through a probabilistic model learned from real data [四]. Zach et al. formulated a TV- $\ell_{1}$ model to estimate flow filed [ $\square$ ]. The $\ell_{1}$ regularization is simple and effective to handle discontinuities. 
To achieve accurate motion information, researchers proposed several parametrized motion models $[\boldsymbol{\square}, \mathbb{\square}]$. Baker and Matthews proposed an unified framework for warping estimation which extends the Lucas-Kanade algorithm [ [ ]]. Employing an over-parametrized motion model, Nir et al. proposed a global energy to estimate motion parameters by extending the Horn-Schunck method [D]. As warping and over-parametrization enlarge the degree of freedom of the motion model, parametrized motion model makes the optic flow estimation inherently go towards the ill-posed problem.

Recently, inspired by the development in compressive sensing [ [ ]], Shen and Wu proposed a sparse model for optic flow estimation [四, $\square$ ]. In this model, the wavelet transformation or the gradient is employed to expose the sparsity of the optic flow. As compressive sensing provides a systematical theory for solving ill-posed problems, the sparse model lays a foundation for optic flow estimation with the convex $\ell_{1}$ optimization. However, when the motion consists rotational and scaling components, both the wavelet and gradient domains are NOT sparse enough, which degrades the solution heavily, in Shen-Wu's model.

In this paper, we propose a novel motion estimation framework by employing parametrized motion model to enhance the sparsity of gradients over motion parameters. Along with making conventional optic flow estimation be more ill-posed, the warping and parametrized models enhance the intrinsic sparsity associated with motion parameters. It is especially apparent for cases that the motion is with rotation and scaling. With this observation, we proposed to tackle the motion estimation problem on the basis of the gradient sparsity of motion parameters. Distinct to Shen and Wu's approach, we formulate the flow estimation as an $\ell_{0}$ optimization problem which provides a deeper perspective about the nature of the problem along with broadened solutions such as the reweighted $\ell_{1}$ minimization [ $\left.\square\right]$. In addition, we relax the constant intensity assumption with an $\ell_{1}$ norm instead of the square error which contributes to the improvement of estimation accuracy.

The rest of this paper is organized as follows. Section 2 describes the parametrized motion model. Section 3 illustrates the sparsity of gradient fields of motion parameters and builds a novel sparse motion estimation framework. The solution to the proposed sparse model is discussed in section 4. Section 5 provides experimental results for evaluating the performance of the proposed method. Section 6 concludes this paper.

\section{Parametrized Motion Model}

Motion is more complex than pure translation. Let $u$ and $v$ be the horizontal and vertical motion components, respectively. Most exiting optic flow estimation approaches are based on this constant motion model. But with such a description, we cannot discover the internal motion structures such as rotation and scaling. Following Nir et al.'s notation [ $\square]$, we employ the following parametrized motion model

$$
\begin{aligned}
& u(x, y)=\sum_{k=1}^{K} p_{k}(x, y) \varphi_{k}(x, y) \\
& v(x, y)=\sum_{k=1}^{K} p_{k}(x, y) \eta_{k}(x, y)
\end{aligned}
$$

to describe the optic flow at a given time instant $t$, where $\varphi_{k}$ and $\eta_{k}$ are basis functions, and $p_{k}$ are corresponding motion parameters. 
Table 1: Summary of parametrized motion models. DOF denotes the number of parameters in the model. The symbol '-' indicates the corresponding parameter is NOT used.

\begin{tabular}{c|c|ccccccc|c}
\hline \multirow{2}{*}{ Motion Model } & & \multicolumn{7}{|c|}{ Parameters } & \multirow{2}{*}{ DOF } \\
\cline { 3 - 8 } & & $p_{1}$ & $p_{2}$ & $p_{3}$ & $p_{4}$ & $p_{5}$ & $p_{6}$ & \\
\hline \multirow{2}{*}{ Constant Motion } & $\varphi_{k}$ & 1 & 0 & - & - & - & - & \multirow{2}{*}{2} \\
& $\eta_{k}$ & 0 & 1 & - & - & - & - & \\
\hline \multirow{2}{*}{ Pure Translation } & $\varphi_{k}$ & 1 & 0 & $x$ & - & - & - & \multirow{2}{*}{3} \\
& $\eta_{k}$ & 0 & 1 & $y$ & - & - & - & \\
\hline \multirow{2}{*}{ Affine Motion } & $\varphi_{k}$ & $x$ & $y$ & 1 & 0 & 0 & 0 & \multirow{2}{*}{6} \\
& $\eta_{k}$ & 0 & 0 & 0 & $x$ & $y$ & 1 & \\
\hline
\end{tabular}

The parametrized model is capable to describe a large set of motion with a diversity of basis functions. If we take 2 parameters with basis functions $\varphi_{1}=1, \varphi_{2}=0, \eta_{1}=0$, and $\eta_{2}=$ 1 , the model degenerates to the conventional constant motion model with $(u, v)=\left(p_{1}, p_{2}\right)$. Increasing the degree of freedom (DOF) of the model, i.e., the number of parameters, we get the pure translation and affine motion models [D], as shown in Table 1.

The pure translation is a special case of general rigid motion limiting to simple translation. The detailed model for a given position $(x, y)$ is

$$
\begin{aligned}
& u=p_{1}+p_{3} x \\
& v=p_{2}+p_{3} y
\end{aligned}
$$

with basis functions in constant motion model augmented with $\varphi_{3}=x$ and $\eta_{3}=y$.

The affine model represents a very general set of motion, including rotation, scaling, and skew. With the basis functions given in Table 1, the affine model is

$$
\begin{aligned}
& u=p_{1} x+p_{2} y+p_{3} \\
& v=p_{4} x+p_{5} y+p_{6}
\end{aligned},
$$

where $(x, y)$ is a given position on the image, and $p_{1}, \cdots, p_{6}$ are affine parameters.

\section{Parametrized Motion Estimation with Sparsity}

To estimate optic flow, we follow the constant intensity assumption [ $\mathrm{\theta}]$ as done in most of existing optical flow estimation methods. When the motion is small, with the first order Taylor expansion, a basic optic flow equation on each pixel $(x, y)$ is derived as

$$
I_{x} u+I_{y} v+I_{t}=0,
$$

where $I_{x}, I_{y}$, and $I_{t}$ are the horizontal, vertical, and temporal partial derivatives of video frame $I(x, y, t)$, and $u=\mathrm{d} x / \mathrm{d} t, v=\mathrm{d} y / \mathrm{d} t$ are the first order approximations of the horizontal and vertical components of the optic flow, respectively.

To solve the under-determined linear system (4), additional constraints are expected. Besides the conventional variational approaches, Shen and $\mathrm{Wu}$ proposed the sparse regularization [四, $\square]$ for optic flow estimation. They assumed both the Haar wavelet decomposition and the gradient of the flow field are sparse. 


\subsection{Sparsity Prior for Motion Estimation}

Following Shen and Wu's work, we assume that the parameter fields of the parametrized motion model (1) are sparse in certain domains. Let the $k$ th parameter field $\boldsymbol{p}_{k}$ denote the $N$ dimensional column vector constructed by stacking corresponding motion parameters over the image, where $N$ is the number of pixels in the image. We suppose the coefficient field $\boldsymbol{g}_{k}$ in domain $\Psi_{k}$ is a sparse signal. Generally, the sparse coefficient field is obtained via a linear transformation

$$
\boldsymbol{g}_{k}=\Psi_{k} \boldsymbol{p}_{k},
$$

where the transformation can be wavelet, DCT, curvelet, gradient, and so many on. If we have the sparse coefficients $\boldsymbol{g}_{k}$, the parameter field can be recovered by

$$
\boldsymbol{p}_{k}=\Psi_{k}^{+} \boldsymbol{g}_{k}
$$

where $\Psi_{k}^{+}$is the inverse or pseudo inverse of $\Psi_{k}$.

Employing the prior knowledge about sparsity, the general framework for parametrized motion estimation is formulated as

$$
\begin{aligned}
& \boldsymbol{p}_{k}^{*}=\underset{\boldsymbol{p}_{k}}{\arg \min _{1}}\|\mathbf{g}\|_{\ell_{0}}=\underset{\boldsymbol{p}_{k}}{\arg \min _{k}}\left\|\boldsymbol{\Psi}_{k} \boldsymbol{p}_{k}\right\|_{\ell_{0}}, \\
& \text { s.t. } \quad I_{x} u+I_{y} v+I_{t}=0 \quad \forall(x, y),
\end{aligned}
$$

where $\mathbf{g}=\left[\boldsymbol{g}_{1}, \cdots, \boldsymbol{g}_{K}\right]$ is the sparse vector field of coefficients, and $\|\cdot\|_{\ell_{0}}$ denotes the so called $\ell_{0}$ norm which counts the number of non-zero values of a signal. The optic flow is recovered with the optimized motion parameters $\boldsymbol{p}_{k}^{*}$ according to the parametrization (1).

\subsection{Sparsity of Motion Parameter Gradients}

With the sparse framework (7), the sparse domain determines the estimation precision. In this paper, we choose the gradients of motion parameters to apply the sparse regularization for motion estimation. The reasons are: a) the gradient of motion parameters are with physical meanings which makes it more intuitive to be understood than wavelet or DCT coefficients, and b) the optic flow field appears strong piecewise discontinuity at object boundaries which are intrinsically coherent with gradients.

Furthermore, the sparsity is enhanced when the DOF of the parametrized motion model increases because the parametrization changes the sparse domain coefficients from piecewise continuous toward piecewise constant. Fig. 1 shows the enhancement of the sparsity of the gradient fields from the constant motion model to the affine motion model. Fig. 1(a) shows the ground truth vectors of a simulated optic flow field with scaling and rotation. Fig. 1(b) shows the horizontal (upper) and vertical (lower) components of conventional constant motion model, and Fig. 1(c) and (d) show the horizontal and vertical gradients of the two motion components, respectively. The horizontal gradients are both sparse while the vertical ones are rather dense due to the rotational motion. Fig. 1(e) shows six motion parameter fields of the affine motion model. Fig. 1(f) and (g) show the horizontal and vertical gradients of all affine parameters, respectively. Gradient fields of affine parameters are all sparser than that of the constant motion model as the former are with non-zero values at only the motion boundary. To make the comparison more clear, Fig. 1(h) and (i) show the gradients as 1D signals for constant and affine motion models, respectively. In addition, Fig. 1(j) shows the 1D signals decomposing the motion field on the Haar wavelet domain. As shown by Fig. 1(h) 
Table 2: The quantitative comparison of the sparsity among Haar wavelet coefficients, gradients of constant and affine motion. The threshold to determine non-zero is set to 0.1 .

\begin{tabular}{rccc}
\hline & Wavelet & Constant Motion & Affine Motion \\
\hline Number of non-zero values & $81.93 \%$ & $75.39 \%$ & $6.08 \%$ \\
Gini-index & 0.7916 & 0.6345 & 0.9694 \\
\hline
\end{tabular}

to $(\mathrm{j})$, the wavelet representation is NOT sparse enough, while the affine gradients is the most sparse representation.

In addition, we make two quantitative comparisons of the sparsity among Haar wavelet coefficients and gradients of the constant and affine motion models. In one comparison, we treat values smaller than a threshold as zeros and others non-zeros. In the other comparison, we compute the Gini index of all signals being compared. According to the comprehensive analysis provided by Hurley and Rickard [四], the Gini index is a good measurement for the sparsity of signals. For the first criteria, the smaller the number of non-zero values is, the sparser the signal is. And for the Gini index, the larger the sparser. From the data shown in Table 2, the degree of sparsity of the wavelet coefficients is equivalent to that of the constant motion model, while the affine motion model shows the strongest sparsity. This certifies that the sparsity is enhanced by employing a parametrized motion model with increased DOF.

\subsection{Gradient Sparsity for Parametrized Motion Estimation}

According to the analysis in previous subsection, we use the sparsity associated with gradients of motion parameters to construct the motion estimation framework. Let $\mathbf{D}_{x, k}$ and $\mathbf{D}_{y, k}$ denote the discrete horizontal and vertical gradient operators, respectively. To estimate motion parameters according to the gradient sparsity, we need to solve the following optimization problem

$$
\begin{aligned}
& \boldsymbol{p}_{k}^{*}=\underset{\boldsymbol{p}_{k}}{\arg \min _{k}} \sum_{k}\left\|\mathbf{D}_{k} \boldsymbol{p}_{k}\right\|_{\ell_{0}}, \\
& \text { s.t. } \quad I_{x} u+I_{y} v+I_{t}=0 \quad \forall(x, y),
\end{aligned}
$$

where $\mathbf{D}_{k}=\left[\mathbf{D}_{x, k}^{T}, \mathbf{D}_{y, k}^{T}\right]^{T}$ denotes the discrete gradient operator with $\mathbf{D}_{k}^{+}$being the pseudo inverse of the operator. The purpose of using different gradient operators for each parameter is to improve the stability and accuracy of the numerical solution, because magnitudes of gradients vary among different motion parameters.

\section{Piecewise Continuous Motion Estimation}

The $\ell_{0}$ problem in the motion estimation models (7) and (8) is a combinatorial optimization which is generally very hard to solve. According to recent advances in compressive sensing $[\boldsymbol{\nabla}]$, if the signal is sparse enough, the problem can be approximately relaxed to a convex $\ell_{1}$ optimization,

$$
\begin{aligned}
& \boldsymbol{p}_{k}^{*}=\underset{\boldsymbol{p}_{k}}{\arg \min _{k}} \sum_{k}\left\|\mathbf{D}_{k} \boldsymbol{p}_{k}\right\|_{\ell_{1}}, \\
& \text { s.t. } \quad I_{x} u+I_{y} v+I_{t}=0 \quad \forall(x, y),
\end{aligned}
$$




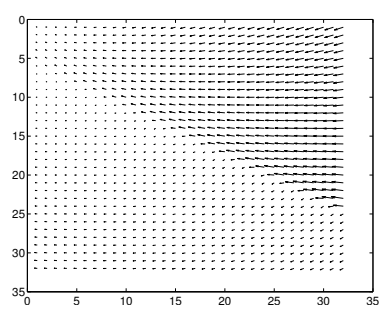

(a)
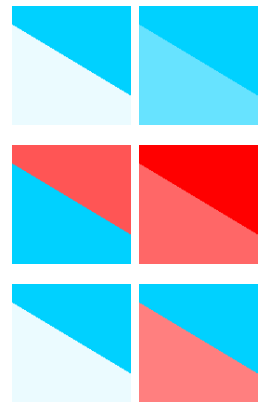

(e)

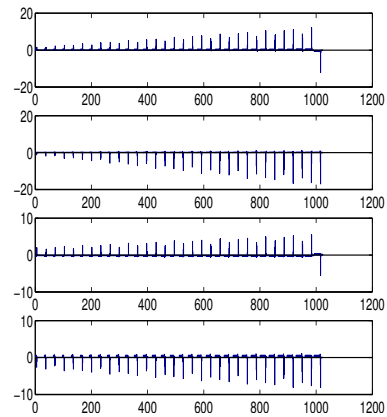

(h)

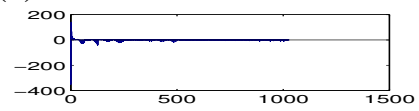

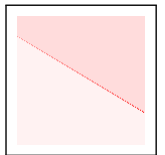

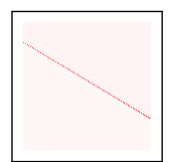

(c)
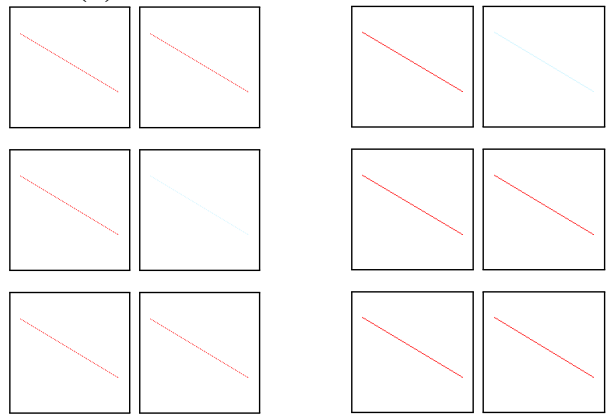

(f)
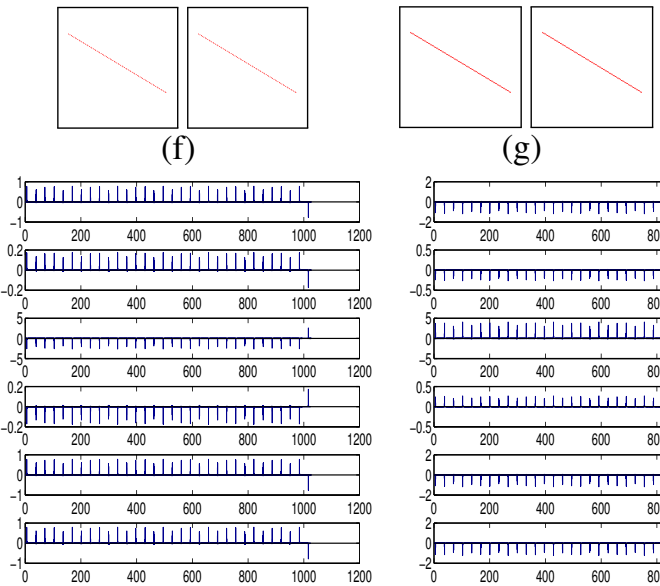

$(\mathrm{g})$

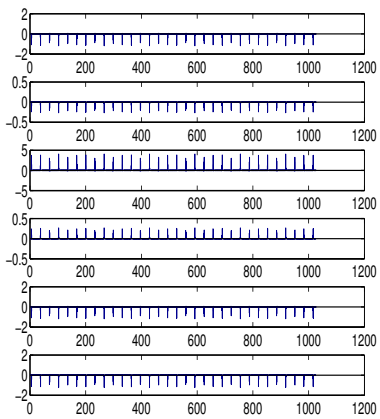

(i)

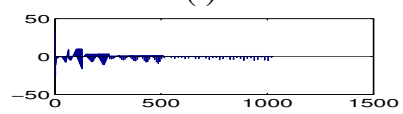

(j)

Figure 1: The comparison of sparsity between gradients of constant and affine motion models. (a) Vector representation of the simulated optic flow field. (b) The horizontal and vertical components of the constant motion. (c) and (d) The horizontal and vertical gradients of both horizontal and vertical components of the constant motion, respectively. (e) The six components of the affine motion. (f) and (g) The horizontal and vertical gradients of all six components of the affine motion, respectively. (h) and (i) The 1D representations of the 2D gradients for constant and affine motion models, respectively. (j) The 1D representation of the Haar wavelet coefficients. 
where $\|\cdot\|_{\ell_{1}}$ denotes the $\ell_{1}$ norm which is the sum of absolute values of all elements of a signal, e.g., $\|s\|=\sum_{i}\left|s_{i}\right|$.

Candès et al. found that model (9) does NOT work well for some cases as it penalizes larger coefficients more heavily than smaller ones [0]. To achieve sparse and accurate solutions, the reweighted $\ell_{1}$ optimization is employed [ $[$ ],

$$
\begin{aligned}
& \boldsymbol{p}_{k}^{*}=\underset{\boldsymbol{p}_{k}}{\arg \min _{k}}\left\|\mathbf{W}_{k} \mathbf{D}_{k} \boldsymbol{p}_{k}\right\|_{\ell_{1}}, \\
& \text { s.t. } \quad I_{x} u+I_{y} v+I_{t}=0 \quad \forall(x, y),
\end{aligned}
$$

where $\mathbf{W}_{k}=\operatorname{diag}\left(w_{k, 1}, \cdots, w_{k, 2 N}\right)$ are the iteratively updated diagonal reweighting matrices. After the $l$-th iteration, the weight for the next iteration is updated with

$$
w_{k, i}^{l+1}=\frac{1}{\left|g_{k, i}^{l}\right|+\varepsilon}
$$

where $\varepsilon$ is a small positive number to avoid dividing by zero.

Usually the constraints of constant intensity assumption (4) cannot hold accurately due to the existence of noise. Shen and $\mathrm{Wu}$ relax the constraints with the $\ell_{2}$ norm [四, 四], or in another name, the least square error criteria. But the $\ell_{2}$ relaxation performs poorly at discontinuities and outliers [G]. On the other hand, the $\ell_{1}$ relaxation is robust on piecewise continuous signals [ [] ] and performs better on motion boundaries. Thus we relax the proposed model (9) and (10) with $\ell_{1}$ norm as

$$
\boldsymbol{p}_{k}^{*}=\arg \min _{\boldsymbol{p}_{k}}\left\{\lambda \sum_{k}\left\|\mathbf{D}_{k} \boldsymbol{p}_{k}\right\|_{\ell_{1}}+\sum_{x, y}\left|I_{x} u+I_{y} v+I_{t}\right|\right\}
$$

and

$$
\boldsymbol{p}_{k}^{*}=\arg \min _{\boldsymbol{p}_{k}}\left\{\lambda \sum_{k}\left\|\mathbf{W}_{k} \mathbf{D}_{k} \boldsymbol{p}_{k}\right\|_{\ell_{1}}+\sum_{x, y}\left|I_{x} u+I_{y} v+I_{t}\right|\right\},
$$

respectively. Both models (12) and (13) are unconstrained convex minimization problems and can be solved efficiently by descent methods [四]. For simplicity, we call models (12) and (13) the ordinary and reweighted $\ell_{1}$ model in the following, respectively. Both models are combined with the constant, pure translations, and affine motion model separately.

\section{Experiments}

In this section, we perform the proposed sparse framework on the Middlebury optic flow benchmark [ $[$ ] and use the standard measures of Average Angular Error (AAE) and End Point Error (EPE) for evaluation. To show the validity and precision, we implement four algorithms, which are Shen and Wu's method [ㅁ] , Nir et al.'s method [ㅁ] , the proposed $\ell_{1}$ norm model (12), and reweighted $\ell_{1}$ norm model (13). Our implementation is based on the $\ell_{1}$-MAGIC toolbox [日].

As the first-order Taylor expansion employed in deriving the basic optic flow equation (4) holds only for small motions, so we downsample all images to $1 / 4$ of their original heights and widths. The downsampled images is divided to $12 \times 12$ overlapping blocks. The proposed models are applied to each image block. For the pixels within overlap areas, the final 
Table 3: The performance comparison on the Middlebury dataset. Shen and Wu's method [ $\square$ ] is denoted as SW. Nir et al.'s method [ $\square$ ] is denoted as Nir, the proposed model (12) with three motion models listed in Table 1, i.e., the constant motion, pure translation, and affine motion, are abbreviated as CGS, PTGS, and AFGS, respectively. While corresponding results of the proposed model (13) are denoted as CGSR, PTGSR, and AFGSR.

\begin{tabular}{r|cccccccc}
\hline & \multicolumn{7}{|c}{ AAE } \\
& SW & Nir & CGS & CGSR & PTGS & PTGSR & AFGS & AFGSR \\
\hline Venus & 6.5389 & 6.6619 & 6.2627 & 5.4088 & 5.6405 & 5.2637 & 5.2454 & $\mathbf{5 . 0 7 5 4}$ \\
Grove2 & 4.1054 & 4.3201 & 3.7880 & 3.6744 & 3.7202 & $\mathbf{3 . 6 4 5 2}$ & 3.7106 & 3.6692 \\
Grove3 & 8.4899 & 8.2010 & 8.0865 & 7.9667 & 7.4611 & 7.4299 & 7.1181 & $\mathbf{7 . 1 1 5 9}$ \\
Dimetrodon & 5.3368 & 5.6604 & 4.6728 & 4.6303 & 4.3994 & $\mathbf{4 . 3 8 9 4}$ & 4.4076 & 4.3971 \\
\hline \hline & \multicolumn{7}{c}{ EPE } \\
& SW & Nir & CGS & CGSR & PTGS & PTGSR & AFGS & AFGSR \\
\hline Venus & 0.1619 & 0.1782 & 0.1543 & 0.1371 & 0.1429 & 0.1352 & 0.1357 & $\mathbf{0 . 1 2 7 3}$ \\
Grove2 & 0.1000 & 0.1057 & 0.0920 & 0.0891 & 0.0900 & $\mathbf{0 . 0 8 8 1}$ & 0.0897 & 0.0888 \\
Grove3 & 0.2759 & 0.2619 & 0.2604 & 0.2567 & 0.2402 & 0.2384 & $\mathbf{0 . 2 2 4 0}$ & 0.2242 \\
Dimetrodon & 0.1101 & 0.1184 & 0.0968 & 0.0961 & 0.0913 & 0.0914 & 0.0914 & $\mathbf{0 . 0 9 1 2}$ \\
\hline
\end{tabular}

flow vector is chosen from all estimated candidates by minimizing the sum of squared difference. To compare the models equally, the RANSAC based refinement scheme [ $\square$ ] and the coarse-to-fine strategy are NOT adopted. For Nir et al.'s method, we perform experiments on their variational approach with the typical 2D affine motion model. The results are obtained on downsampled images without using the coarse-to-fine strategy. Shen and Wu's method also performs the down-sampling scheme employed in our method.

As the magnitude of gradients varies with respect to the motion parameter, we use different kernels to build the discrete gradient operators. For constant motion, we use kernels $[1,-1]$ and $[1,-1]^{T}$ to build $\mathbf{D}_{1}$ and $\mathbf{D}_{2}$. For pure translation, we build $\mathbf{D}_{3}$ with kernels $[100,-100]$ and $[100,-100]^{T}$ while use kernels $[1,-1]$ and $[1,-1]^{T}$ to build $\mathbf{D}_{1}$ and $\mathbf{D}_{2}$. For the affine motion, we use kernels $[1,-1]$ and $[1,-1]^{T}$ to build $\mathbf{D}_{3}$ and $\mathbf{D}_{6}$, kernels $[100,-100]$ and $[100,-100]^{T}$ to build $\mathbf{D}_{1}, \mathbf{D}_{2}, \mathbf{D}_{4}$, and $\mathbf{D}_{5}$. For other parameters, we set $\lambda=1.5$ in Shen and Wu's method, $\lambda=11$ in Nir et al.'s method. In both proposed models, we use a same $\lambda$ for same motion model. More specifically, We set $\lambda=3.3, \lambda=8$, and $\lambda=18$ for constant motion, pure translation, and affine motion, respectively.

The AAE and EPE of the methods on four test sequences are listed in Table 3. For both criteria and all 4 test sequences, our models all perform better than both methods proposed by Shen-Wu and Nir et al. This shows our sparsity model is suitable for piecewise continuous optic flow estimation. Comparing the SW and CGS columns, our model provides more accurate results than Shen-Wu's method. With same motion model, the optic flow fields recovered by the reweighted $\ell_{1}$ model (13) is better than that by the ordinary $\ell_{1}$ model (12), except the EPE on Grove3 with affine motion. To sum up, both proposed models perform better and better when the DOF of motion model increases. This certifies that the parametrization of motion model improves the motion estimation accuracy.

A special case in Table 3 is that, on Grove 2 and Dimetrodon, the pure translation model with reweighted $\ell_{1}$ scheme performs better than affine motion model with ordinary $\ell_{1}$ scheme. This is because for certain regions of the motion field, pure translational motion is fine enough. And further more, though it enhances the sparsity, the affine model also enlarge the dimensionality of the problem to be solved which degrades the solution quality.

Some results of the tested optic flow estimation methods are illustrated in Fig. 2. From 


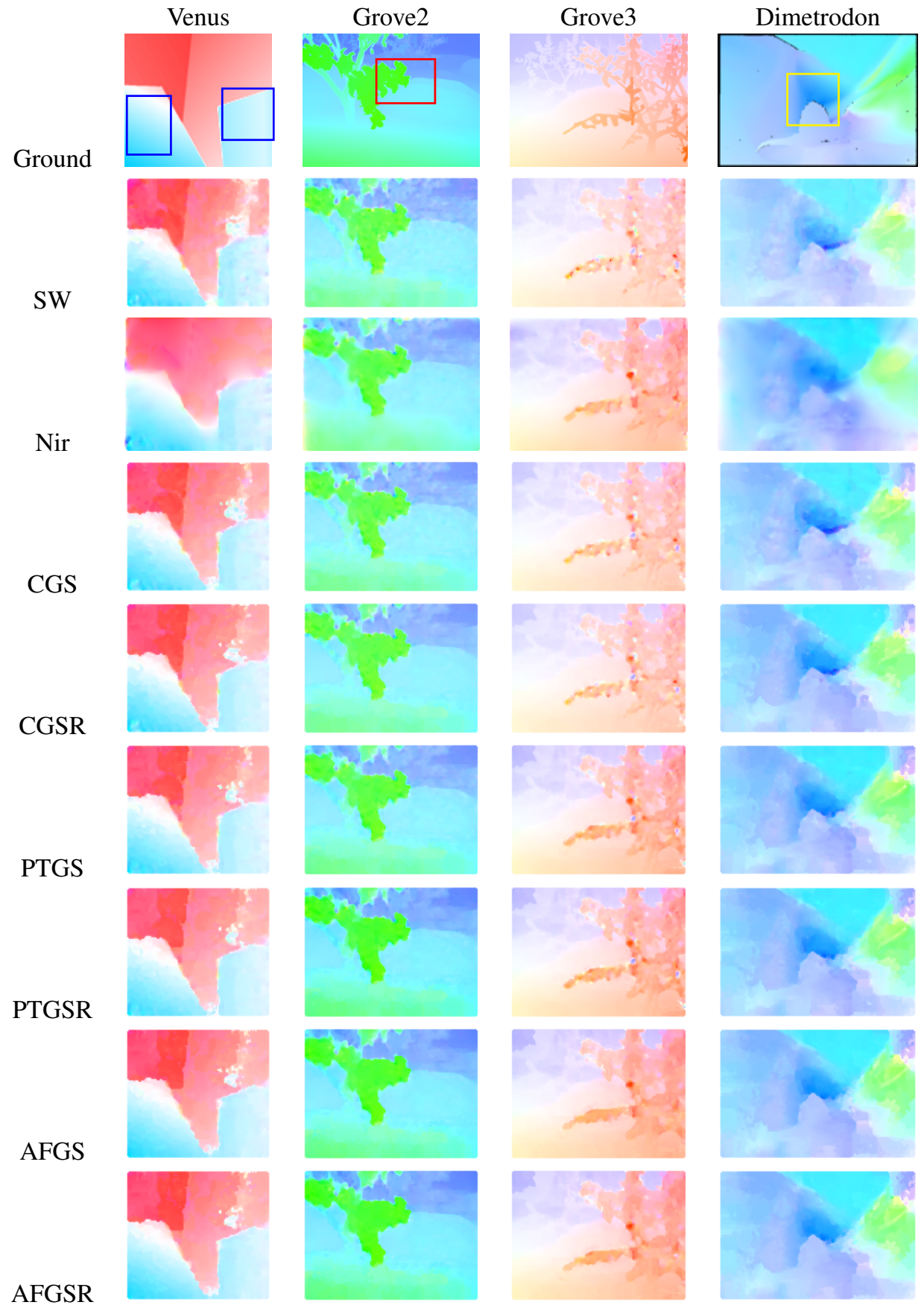

Figure 2: The result optic flow fields. From top to bottom, the first three rows show the Ground truth, results of Shen and Wu's method[ㅁ] ], and results of the Nir et al.'s method[ㅁ] ]. The rest rows show results of the proposed ordinary and reweighted $\ell_{1}$ models alternately, with constant motion, pure translation, and affine motion in turn. 
left to right, are the four test cases, which are Venus, Grove2, Grove3, and Dimetrodon, respectively. The optic flow regions with gradual changes become better and better from top to bottom. These regions are marked out by color boxes on ground truth. In the Venus sequence, the proposed method achieves more crisp motion boundaries and/or more smooth motion field (regions with affine motion) than Shen-Wu's method in both blue boxes. Similar results can also be found in the red and yellow rectangles marked out in Grove 2 and Dimetrodon sequences.

\section{Conclusions}

In this paper, we generalize the sparsity regularized optic flow estimation to parametrized motion model. From the perspective of sparse signal recovery, we formulate the flow estimation as an $\ell_{0}$ optimization. By exploiting the gradient sparsity of parametrized motion model, this problem is relaxed to an $\ell_{1}$ optimization. Along with $\ell_{1}$ norm regularization, the optimization is reduced to unconstrained $\ell_{1}$ minimization. Experiments certify our arguments on increasing the DOF of motion model enhances the sparsity of motion parameter gradients and hence produces robust and accurate motion estimation results. In addition, the reweighted optimization scheme contributes strongly to the improvement of accuracy.

In Shen and Wu's work [ $\square$ ], a robust refinement strategy is proposed to achieve more accurate estimation. This strategy is feasible in our method too. As the basic optic flow equation holds on small motion only, the proposed method is only work on down-sampled images. To estimate large motion on original images, coarse-to-fine strategy is expected.

\section{Acknowledgments}

This work is supported by National Natural Science Foundation of China under grant number 60805012, 61070138, and 60736043, and the Fundamental Research Funds for the Central Universities under grant number JY10000902006.

\section{References}

[1] S. Baker, D. Scharstein, J. P. Lewis, S. Roth, M. J. Black, and R. Szeliski. A database and evaluation methodology for optical flow. Int. J. Computer Vision, 92:1-31, 2011.

[2] Simon Baker and Iain Matthews. Lucas-kanade 20 years on: a unifying framework. Int. J. Computer Vision, 56:221-255, 2004.

[3] M.J. Black and P. Anandan. A framework for the robust estimation of optical flow. In Int'l Conf. Computer Vision, pages 231-236, 1993.

[4] Stephen Boyd and Lieven Vandenberghe. Convex Optimization. Cambridge University Press, 2004.

[5] Andrés Bruhn, Joachim Weickert, and Christoph Schnörr. Lucas/Kanade meets Horn/Schunck: combining local and global optic flow methods. Int. J. Computer Vision, 61(3):1-21, 2005. 
[6] E. Candès and J. Romberg. $\ell_{1}$-MAGIC. Available online, 2005. URL http: //users.ece.gatech.edu/ justin/llmagic/.

[7] E. Candès, M. Wakin, and S. Boyd. Enhancing sparsity by reweighted $\ell_{1}$ minimization. J. Fourier Analysis and Applications, 14(5):877-905, December 2008.

[8] D.L. Donoho. Compressed sensing. IEEE Trans. Inf. Theory, 52(4):1289-1306, 2006.

[9] B. K.P. Horn and B. G. Schunck. Determining optical flow. Artificial Intelligence, 17 (1-3):185-203, August 1981.

[10] N. Hurley and S. Rickard. Comparing measures of sparsity. IEEE Trans. Inf. Theory, 55(10):4723-4741, 2009.

[11] B. D. Lucas and T. Kanade. An iterative image registration technique with an application to stereo vision. In Proc. 7th Int'l Joint Conf. Artificial Intelligence, volume 2, pages 674-679, Vancouver, BC, Canada, 1981.

[12] T. Nir, A.M. Bruckstein, and R Kimmel. Over-parameterized variational optical flow. Int. J. Computer Vision, pages 205-216, 2008.

[13] Nils Papenberg, Andrés Bruhn, Thomas Brox, Stephan Didas, and Joachim Weickert. Highly accurate optic flow computation with theoretically justified warping. Int. J. Computer Vision, 67(2):141-158, 2006.

[14] Xiaohui Shen and Ying Wu. Exploiting sparsity in dense optical flow. In 17th IEEE Int'l Conf. Image Processing (ICIP),, pages 741-744, 2010.

[15] Xiaohui Shen and Ying Wu. Sparsity model for robust optical flow estimation at motion discontinuities. In IEEE Conf. Computer Vision and Pattern Recognition, CVPR, pages 2456-2463, 2010.

[16] Deqing Sun, S. Roth, J.P. Lewis, and M.J. Black. Learning optical flow. In European Conf. Computer Vision, ECCV, volume 5304 of LNCS, pages 83-97. Springer-Verlag, 2008.

[17] C. Zach, T. Pock, and H. Bischof. A duality based approach for realtime TV- $\ell_{1}$ optical flow. In F. Hamprecht, C. Schnörr, and B. Jähne, editors, Pattern Recognition, DAGM 2007, volume 4713 of $L N C S$, pages 214-223. Springer, 2007. 